\title{
Enhancement of Advanced Range Telemetry (ARTM) Channels via Blind Equalization
}

\author{
Zhong Ye, Edgar H. Satorius and Thomas C. Jedrey \\ Jet Propulsion Laboratory \\ California Institute of Technology \\ 4800 Oak Grove Drive \\ Pasadena, California 91109
}

\author{
Kip Temple \\ 412th Test Wing, Air Force Test Center \\ Edwards A.F.B.
}

\begin{abstract}
The Joint Services Advanced Range Telemetry (ARTM) Program at Edwards Air Force Base has been evaluating FQPSK-B for possible upgrades to the existing telemetry equipment. It has been found in the wideband channel sounding experiments sponsored by ARTM that the in-flight fading channel can be modeled as a 3-ray multipath channel ${ }^{[1]}$. Delay spread for a typical in-flight channel is in the order of 300 nanoseconds. Furthermore, the pre-flight channel is characterized by much more severe multipath, in which the delay spread is in the order of microseconds covering one or more symbols when the FQPSK-B transceiver operates at a rate of millions of symbols per second. This adverse channel condition inevitably causes tremendous distortion in the received signals due to severe inter-symbol interference (ISI) from the multipath.
\end{abstract}

This paper provides an assessment of the potential ability of blind equalization to reduce the FQPSK-B system susceptibility to degradation caused by dynamic frequency selective fading in the aeronautical telemetry environment. In particular, a blind equalizer applique that can be inserted prior to the demodulator without knowledge of the received signal such as carrier frequency, symbol timing and sequence, etc, is proposed. Since it is desired that the equalizer applique operate independently of the carrier frequency and given that the modulation of interest is constant envelope (PCM-FM or FQPSK$\mathrm{B})$, we have selected the constant modulus algorithm $(\mathrm{CMA})^{[2]}$ cost function for implementation. Extensive tests on both simulated and recorded FQPSK-B data transmitted over different ARTM channels have been conducted and the blind equalizer structure has shown substantial improvements, even on the difficult ARTM pre-flight channels. The CMA adapts the equalizer coefficients to minimize the deviation of the output envelope from an arbitrary constant level.

This paper depicts the pre-flight and in-flight channel conditions using time and spectral domain measurement. It quantifies the benefit of the blind CMA tapped delay line equalizer. Due to the extensive signal processing requirements associated with the very high sampling rate $(100 \mathrm{MHz})$ of the FQPSK-B system, hardware implementation complexity is very high. Complexity reduction issues regarding the implementation of the CMA using Field Programmable Gate Array (FPGA) will also be presented. 


\section{INTRODUCTION}

In an attempt to quantify any improvements provided by blind equalization for the ARTM channels, extensive tests have been conducted on both simulated and recorded ARTM channel data corresponding to FQPSK-B modulated data. The intent of this work is to establish the feasibility of a blind equalizer applique that can be inserted prior to the demodulator without knowledge of either the signal carrier frequency or symbol timing information. As such, the equalizer can operate either at the digital IF frequency or at complex baseband. The latter mode is chosen for our testing purposes as described in Section 2 of this paper.

Since it is desired that the equalizer applique operate independently of the carrier frequency and given that the modulation of interest is constant envelope (PCM-FM or FQPSK-B), we have selected the constant modulus algorithm (CMA) cost function for implementation and testing. The CMA adapts the equalizer coefficients to minimize the deviation of the output envelope from an arbitrary constant level, chosen to be unity for our testing. As will be demonstrated here, the CMA yields excellent improvement even on the difficult, ARTM pre-flight channels. Although the CMA equalization results reported herein use a tapped delay line digital filter (non-baud spaced), other filter realizations (e.g., lattice, recursive) are currently being developed and tested.

Given that the pre-flight channels are generally the most difficult to demodulate due to the ground multipath, our initial testing has focused on these data segments. Preliminary evaluation of the in-flight data reveals that severe fading occurs only for a slight fraction of the entire recording period. For most of the other time, channel impairment is so little that equalization provides no measurable improvement in the output bit error rate (BER). When the fading and/or co-channel interference event does occur, preliminary results indicate BER improvement from the equalizer. hi this paper, processing results of the sampled IF ground data for the ARTM Flight measurement data are presented. The paper is organized into the following sections:

(1) Summary of the ARTM Flight 21 data points.

(2) Description of the data processing steps.

(3) Equalizer structure

(4) Processing results from the data set

(5) Complexity reduction

(6) Hardware implementation issues

\section{ARTM FLIGHT 21}

The test aircraft was configured with two omnidirectional transmit antennas in a top and bottom configuration with equal power being applied to each antenna. The phase relation between the two TX antennas is fixed (but unknown). This antenna configuration was chosen because it is the standard for flight test aircraft thought to minimize telemetry dropouts due to the aircraft shading the receive antenna. It does though create co-channel interference. Antenna nulls that may not normally exist with just one onmidirectional antenna on the aircraft are artificially created.

For this test the modulation is FQPSK-B at a bit rate of $14.7 \mathrm{Mb} / \mathrm{s}(7.35 \mathrm{Msymbols} / \mathrm{sec})$ with the transmit frequency of $2374.5 \mathrm{MHz}$. The receive system consisted of a 4 foot parabolic dish antenna, low noise amplifier, telemetry receiver with front-end fast automatic gain control, and a FQPSK-B demodulator. 
The collected data is the received intermediate frequency (IF) at $70 \mathrm{MHz}$ sampled at $100 \mathrm{MHz}$. Thus the center of the passband is folded (aliased) down to $30 \mathrm{MHz}$ and inverted on the frequency axis. Each sample is 8 bits in length.

There are two types of channel conditions captured during the test, preflight and airborne. For the preflight condition, the aircraft moved slowly (taxied) on the ramp while snapshots of the IF were taken. The airborne (in-flight) condition consisted of flying through known areas of multipath and sampling the IF at those points.

The RF channel is characterized by the following two impairments:

- Multipath propagation, primarily manifested as time varying frequency selective fading;

- Co-channel interference due to the dual TX antennas.

\section{DESCRIPTION OF DATA PROCESSING STEPS}

Figure 3-1 is a block diagram describing the intermediate processing steps for the received IF sampled data:

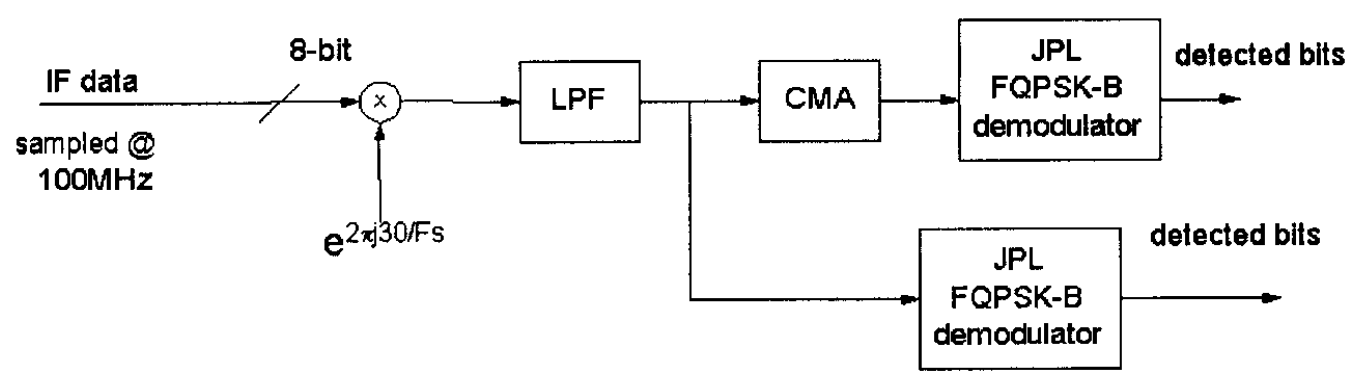

Figure 3- 1 Sampled IF data processing

(1) The real sampled IF data, centered at $30 \mathrm{MHz}$, is first translated to complex baseband by mixing with the $30 \mathrm{MHz}$ complex carrier.

(2) The down-converted signal is further low-pass filtered with a cutoff frequency of $15 \mathrm{MHz}$, corresponding to the main-lobe of the spectrum.

(3) Note that all of these operation are performed asynchronously, i.e., no symbol timing information is required.

(4) The complex baseband data are processed along two parallel paths. For the un-equalized path, data passes through the FQPSK-B software demodulator. BER analysis is then performed using crosscorrelation against the known transmitted bit sequence, which is a $\left(21^{11}-1\right)$ PN sequence.

(5) For the equalized path, the data first passes through the CMA blind equalizer. The equalized data then follows the same processing as described above in Item (4).

Throughout the data processing, the time domain signal constellation, histograms and spectra of selected data segments are computed and displayed to characterize the ARTM channel. Figure 3-2 shows these for illustration. They were obtained from the perfect received signal contained in the Flight CD. This represents example of a "clear" channel without apparent multipath. 

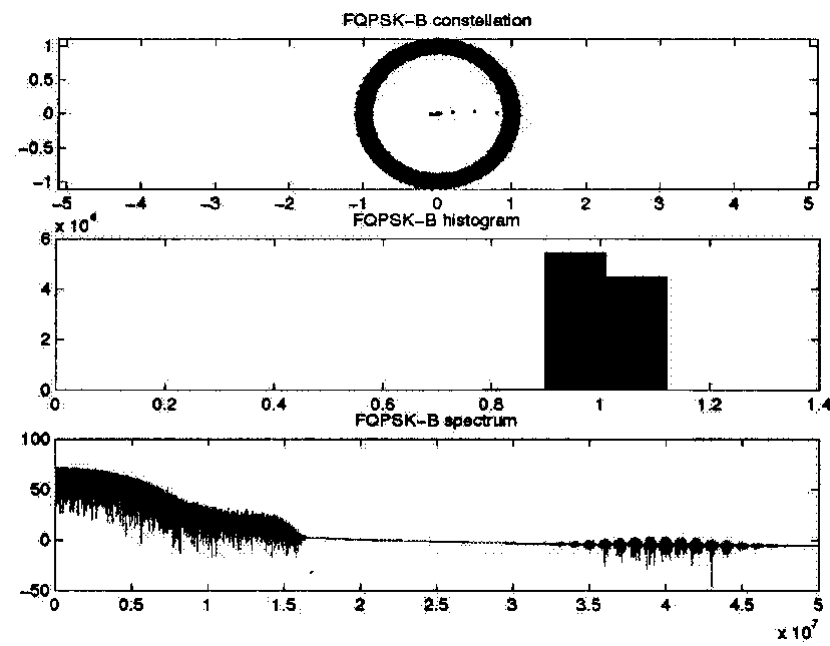

Figure 3-2 FQPSK signal characteristics

\section{EQUALIZER}

Figure 4-1 shows the structure of a CMA, tapped delay line blind equalizer ${ }^{[1]}$.

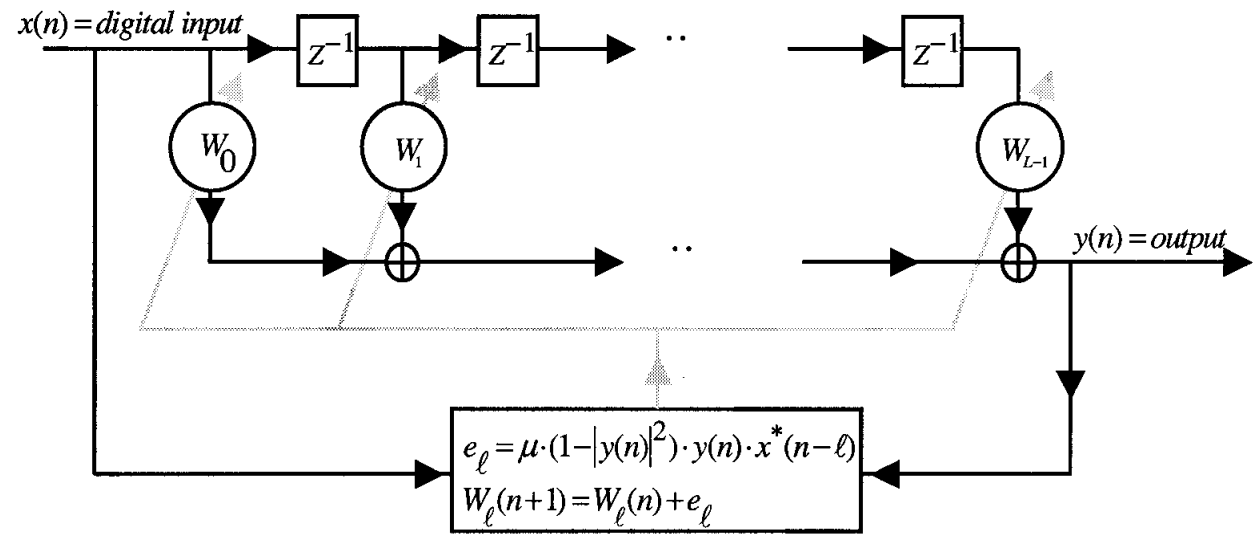

Figure 4-1 Tapped delay line CMA equalizer

In essence, the CMA seeks to minimize a cost function defined by the constant modulus (CM) criterion. The CM criterion penalizes deviations in the magnitude of the equalized signal from a fixed value. The stochastic gradient descent algorithm is used to minimize the CM cost function, starting at an initial, non-zero tapped delay line weight vector corresponding to the symmetric, all-pass filter. In summary, the equalizer:

- Maintains unity output envelope, i.e., $|\mathrm{y}(\mathrm{n})|^{2} \sim 1$, even in multipath distortion

- Can operate asynchronously with the data clock and in the presence of carrier/Doppler offset, which makes it an ideal applique candidate

The number of equalizer taps, $\mathrm{L}$, depends on the multipath and data rate -- for a given multipath profile $\mathrm{L}$ decreases with increasing data rate. In addition, the CMA convergence rate is determined by $\mathrm{L}$ and $\square$, the convergence step size. Based on simulations performed to date, the worst-case settling time is bounded by 100,000 symbols. 
For all of the data processing tests, $\mathrm{L}$ is fixed at 1000 taps for the pre-flight channel, and $\mathrm{L}=100$ for the in-flight channels. From Figure 4-1, the operation complexity of the tapped delay line CMA equalizer can be estimated. Assuming the length of this fractional spaced equalizer to be L, there will be approximately 2L MULTIPLIES and 2L ADDS in every clock cycle. Given that the sampling frequency is $100 \mathrm{MHz}$, the total number of MULTIPLIES and ADDs is $200 \mathrm{~L}^{*} 10^{6}$ per second and $200 \mathrm{~L} * 10^{6}$ per second respectively. Given that the algorithm complexity is linearly proportional to L, subsequent data processing with the tapped delay line CMA equalizer will attempt to reduce the implementation complexity via the following:

- Reduce L

- Incorporate decimation prior to the equalizer

In addition, other filter realizations (e.g., lattice, recursive $)^{[3]}$ are currently being developed and tested, aiming to reduce the implementation complexity. In summary, the structure of the add-on equalizer module is shown in Figure 4-2. It represents an applique solution to the current receiver.

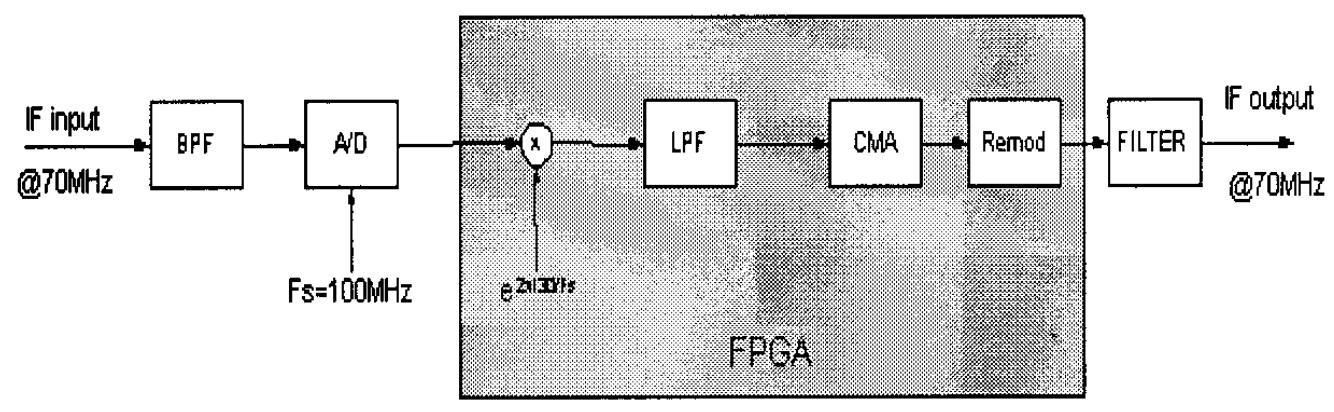

Figure 4-2 Structure of add-on equalizer

\section{RESULTS}

\subsection{PRE-FLIGHT CHANNEL}

Figure 5-1 is the decimated average power plot for a typical preflight channel. The temporal behavior of the plot has a good indication of the time varying fading characteristics of the in-flight channel.

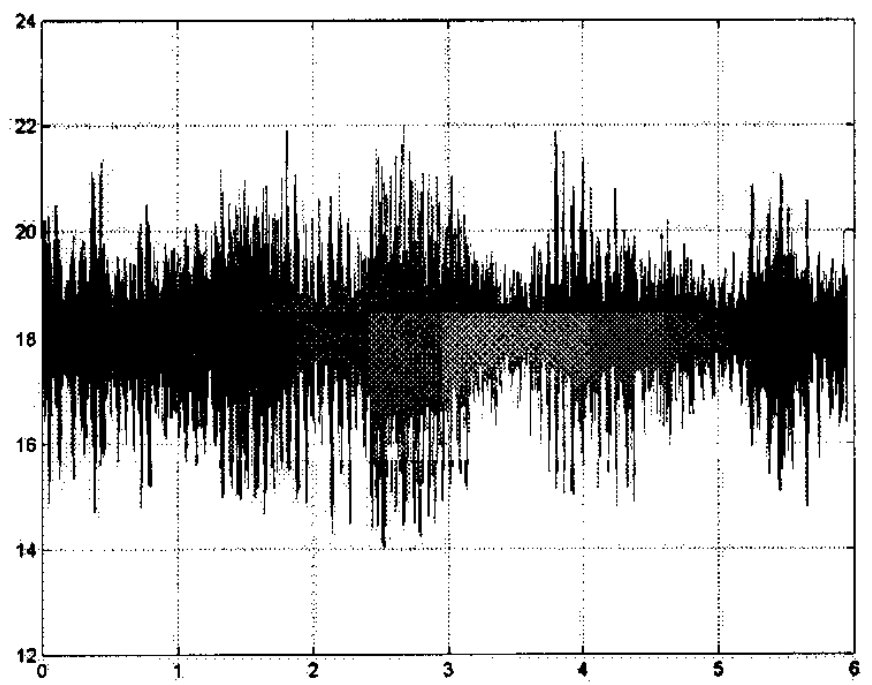

Figure 5-1 Pre-flight channel decimated average power 
Refer to Figure 5-2 through 5-4 for the corresponding signal constellation, histogram and spectrum plots. These plots indicate how bad the channel impairment is in time, frequency domain. They also reflect the effectiveness of the equalizer. Table 5-1 lists the BER performance obtained from processing the fading segments of the collected data. Note that the equalizer length is kept to 1000 for these preflight channels.
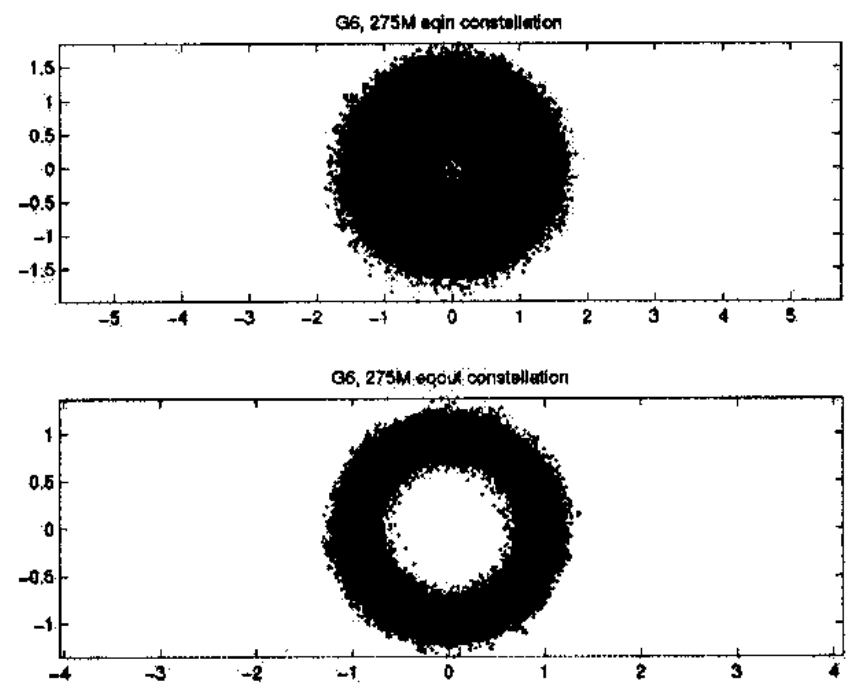

Figure 5-2 Signal constellation of G6 channel Segment A
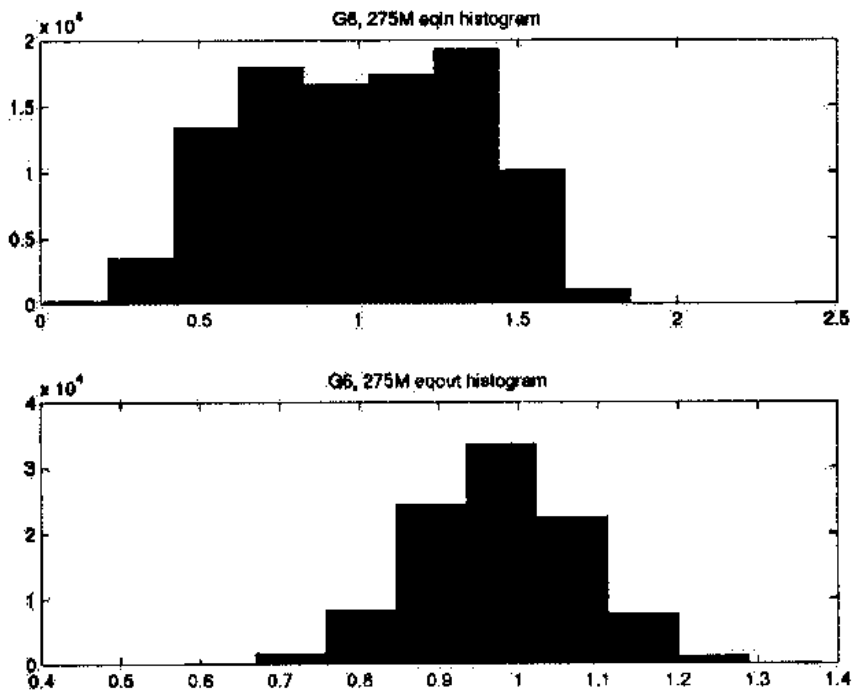

Figure 5-3 Histogram of G6 channel Segment A 

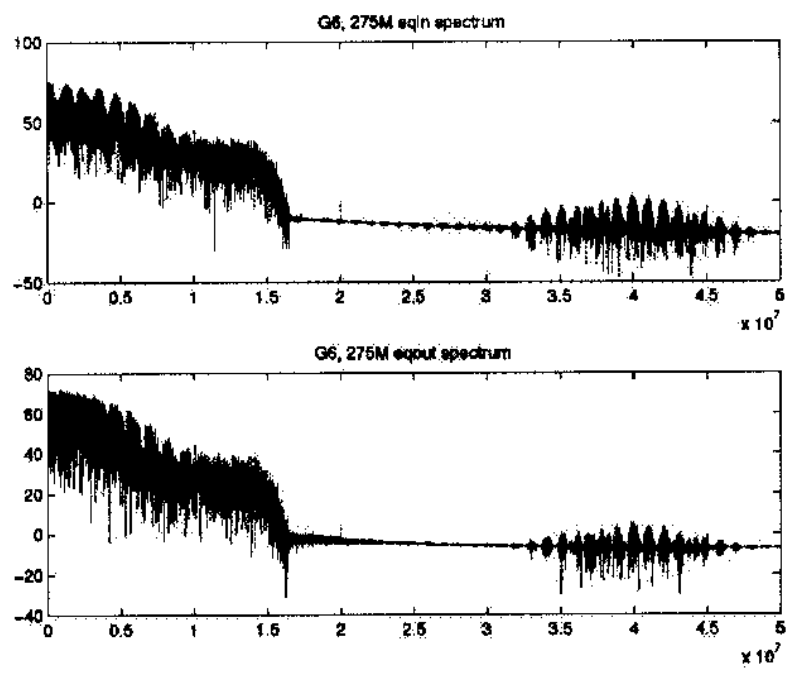

Figure 5-4 Signal spectrum of G6 channel Segment A

Table 5-1 BER performance for pre-flight channel

\begin{tabular}{|c|c|c|}
\hline $\begin{array}{c}\text { fading } \\
\text { segment }\end{array}$ & $\begin{array}{c}\text { BER without } \\
\text { equalization }\end{array}$ & BER with CMA \\
\hline 1 & 0.460 & $8.0 \mathrm{e}-3$ \\
\hline 2 & $3.0 \mathrm{e}-3$ & $2.5 \mathrm{e}-4$ \\
\hline
\end{tabular}

Figure 5-5 shows the modulus error plot for the equalizer. As is seen, the equalizer requires approximately 100000 samples for convergence in this case.

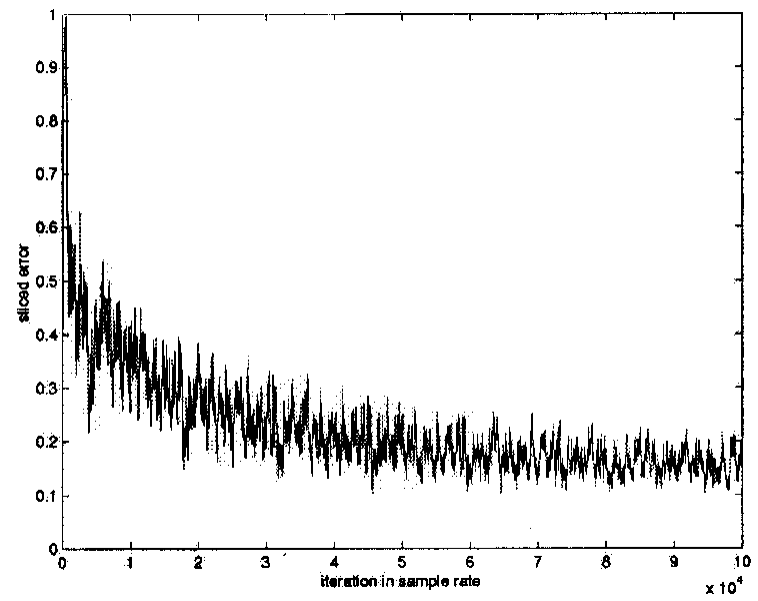

Figure 5-5 CMA equalizer convergence behavior, Segment A of G6 channel

\subsection{IN-FLIGHT CHANNEL}

Equalization of the in-flight channel substantially improves the BER performance as indicated by Table 5-2. Note that the equalizer length is kept to 100 for the in-flight channel. 
Table 5-2 BER performance for in-flight channel

\begin{tabular}{|c|c|c|}
\hline fading segment & $\begin{array}{c}\text { BER without } \\
\text { equalization }\end{array}$ & BER with CMA \\
\hline 1 & $3.8 \mathrm{e}-3$ & $4.5 \mathrm{e}-4$ \\
\hline 2 & $2.76 \mathrm{e}-5$ & $2.8 \mathrm{e}-5$ \\
\hline
\end{tabular}

\section{COMPLEXITY REDUCTION}

In the following, we compare the BER performance of the regular CMA with those of the decimated counterpart. Because the pre-launch channel requires much longer equalizer length than the in-flight channel, the preliminary evaluation operates only on two of the pre-launch channels, G4 and G6, as indicate din Table 6-1 and 6-2. Decimation factor is kept to 5.

Table 6-1 Decimation Performance for G4 channel

\begin{tabular}{|c|c|c|c|}
\hline segment & $\begin{array}{c}\text { BER without } \\
\text { equalizer }\end{array}$ & $\begin{array}{c}\text { BER with } \\
\text { regular } \\
\text { CMA }\end{array}$ & $\begin{array}{c}\text { BER with } \\
\text { decimated } \\
\text { CMA }\end{array}$ \\
\hline 1 & 0.452 & $2.91 \mathrm{e}-3$ & $5.8 \mathrm{e}-3$ \\
\hline 2 & 0.480 & $1.0 \mathrm{e}-2$ & $1.26 \mathrm{e}-2$ \\
\hline 3 & 0.295 & $1.0 \mathrm{e}-5$ & $8.33 \mathrm{e}-4$ \\
\hline 4 & $3.73 \mathrm{e}-3$ & $1.0 \mathrm{e}-5$ & $1.0 \mathrm{e}-5$ \\
\hline
\end{tabular}

Table 6-2 Decimation Performance for G6 channel

\begin{tabular}{|c|c|c|c|}
\hline segment & $\begin{array}{c}\text { BER without } \\
\text { equalizer }\end{array}$ & $\begin{array}{c}\text { BER with } \\
\text { regular } \\
\text { CMA }\end{array}$ & $\begin{array}{c}\text { BER with } \\
\text { decimated } \\
\text { CMA }\end{array}$ \\
\hline 1 & 0.48 & 0.40 & $3.31 \mathrm{e}-2$ \\
\hline 2 & 0.49 & $8.57 \mathrm{e}-3$ & $5.49 \mathrm{e}-3$ \\
\hline 3 & $8.8 \mathrm{e}-3$ & $3.63 \mathrm{e}-3$ & $3.70 \mathrm{e}-3$ \\
\hline 4 & $1.43 \mathrm{e}-1$ & $1.0 \mathrm{e}-5$ & $1.0 \mathrm{e}-5$ \\
\hline
\end{tabular}

The decimation factor of 5 reduces the overall complexity by a factor of $5^{2}$, or 25 . In majority of the cases, the decimated system shows comparable performance to the non-decimated system. The fact that the decimated system shows even slightly better performance in some cases indicates that the channel delay spread may not be as bad as originally anticipated, which requires a 1000-taps equalizer.

\section{HARDWARE IMPLEMENTATION ISSUES}

The structure of the add-on equalizer module has been shown in Figure 4-2. It represents an applique solution to the current receiver. In the following, we discuss some of the hardware implementation issues, aiming to fit the CMA algorithm into the current available Field Programmable Gate Array (FPGA) devices.

The conventional CMA equalizer adjusts the equalizer coefficients for every input symbols. Furthermore, the fractionally spaced CMA equalizer presented earlier operates at a fraction of the symbol rate. Thus the computational complexity becomes higher in proportional to the sampling 
frequency. In order to reduce the cost required for the high speed hardware, the block adaptation algorithm can be employed and its block diagram is shown in Figure 7-1. It is well known that the block adaptation algorithm shows comparable performance as conventional algorithm, if the input signals do not vary so rapidly ${ }^{[4]}$. By using the block CMA and the fast convolution in the DFT domain, the computations for the CMA can be further reduced. Similar schemes have been developed for the linear filtering, that is, the frequency domain adaptive filter has been applied for the linear adaptive filtering applications $^{[5]}$. The approach employs fast linear convolution in the DFT domain using overlap and save method as illustrated in Figure 7-2.

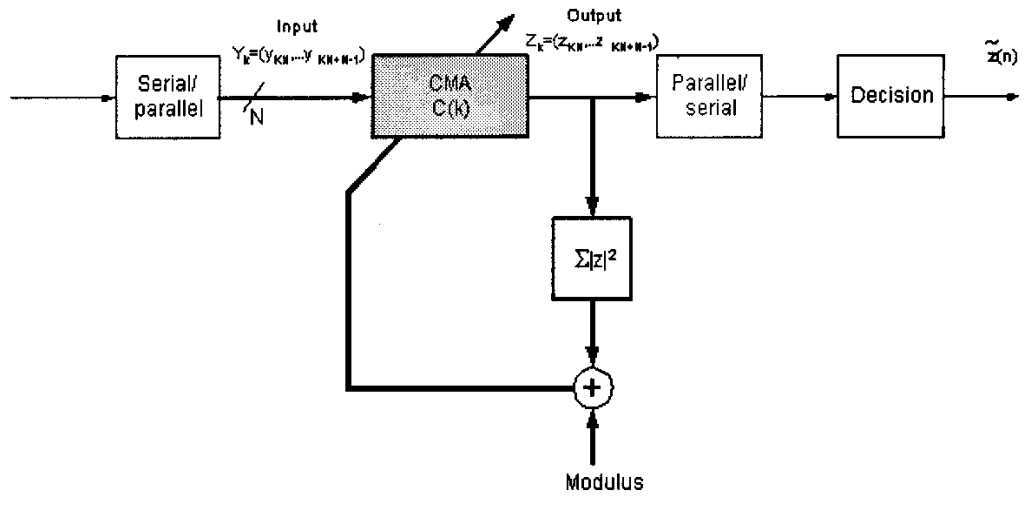

Figure 7.1 Block CMA

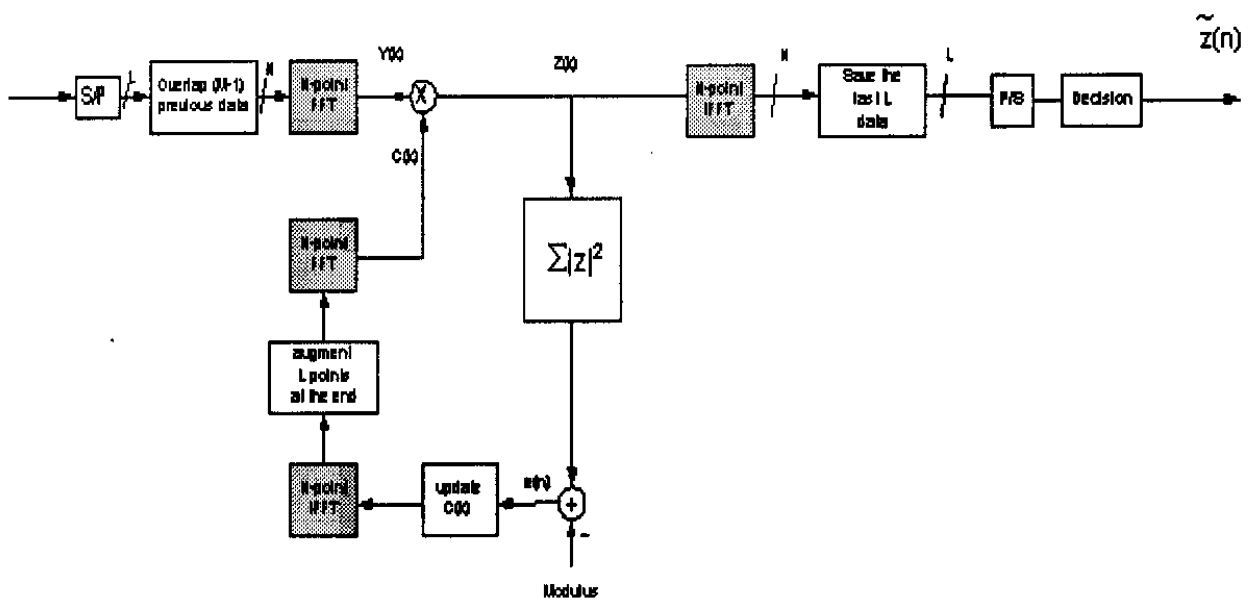

Figure 7.2 Frequency Domain Block CMA

The main advantage of frequency domain block CMA is the saving in computations. If the block size and filter length is $\mathrm{N}$, the multiplications required for the conventional CMA and FBCMA is in the order of $\mathrm{O}\left(N^{2}\right)$ and $\mathrm{O}\left(\operatorname{Nlog}_{2} N\right)$ respectively ${ }^{[6]}$. For a 256-tap FIR implementation, the authors showed that about 20 times less complexity for FBCMA with comparable performance could be achieved. In addition, the frequency domain filtering operation is suitable for pipelined implementation using FPGA devices $^{[7,8]}$.

As just discussed, to double the throughput of a FIR filter without increase the clock rate of the filter itself, the filter area would have to be doubled. I.e., two polyphase decompositions of the filter would be created, one containing the even samples of the original filter, the other the odd. The same concept can be extended such that the overall filter is decomposed into a sum of $N=2^{m}$ sum-filters, each of which 
filters a particular "phase" of the input signal. This polyphase structure is suitable for FPGA implementation for both decimation and interpolation filter. This is critical for the decimated CMA operation.

Optimal design can be achieved by a more careful examining of the FPGA structure. One of the important features in the XILINX architecture is the ability to use configurable logic blocks (CLB) Look-up tables (LUTs) as RAM. Each LUT can be, used as a 16x1 RAM. There are two LUTs in each CLB, allowing a single CLB to either implement a 32x1, a 16x2 or two 16x1 RAMs with a shared clock[9]. The abundance of small distributed RAM blocks throughout the chip enables the user to precalculate partial products, and to load these into the distributed RAM, thereby eliminating the large amounts of logic needed to compute multiplication results in a non-distributed approach. The most versatile approach to using the distributed RAM is an approach called Distributed Arithmetic (DA) $[10,11,12,13]$. Using Xilinx FPGA design tools called Core Generator, a lot of signal processing algorithm can be efficiently implemented using distributed algorithm.

\section{CONCLUSIONS}

Following is a summary of the ARTM data processing:

- Provided the channel CNR is adequate the CMA tapped delay line equalizer can effectively mitigate the effects of the severe multipath in the pre-flight channels.

- The CMA equalizer is also effective to mitigate in-flight channel impairments and furthermore does not degrade demodulator performance under clear flight conditions.

- The pre-flight channel can be modeled as a slowly time varying, severe frequency selective fading channel.

- The in-flight channel is characterized by long intervals of little or no multipath distortion (flat fading conditions) interspersed with short but severe CCI due to the dual TX antennas. Time variation is faster than for the pre-flight channel.

- Under moderate CNR condition and when the constellation is almost close, the CMA equalizer is very effective in enhancing the noise immunity of the system by opening up the constellation.

- When the channel CNR is low, the CMA equalizer can not substantially improve BER performance. This is a fundamental limitation of any equalizer structure (tapped delay line or recursive) operating under very low CNR conditions.

- The only strategies that can be effectively applied to low CNR channel conditions include forward error correction (FEC); automatic re-transmission request (ARQ) protocols; adaptive modulation and antenna diversity - techniques that are well outside the realm of an applique.

- Experiments with multirate processing reveals that the equalizer complexity can be reduced by an order of magnitude without compromising performance. This is especially critical for pre-flight processing and essentially enable the FPGA implementation of a realtime equalizer applique.

- Further complexity reduction can be obtained through parallel processing techniques including polyphase filtering and frequency domain adaptive processing.

\section{REFERENCES}

[1] Michael Rice, David de Gaston, Adam Davis, Gus German, and Chris Bettwieser "ARTM Channel Sounding Results -- An hivestigation of Frequency Selective Fading on Aeronautical Telemetry Channels", Proceedings of the International Telemetering Conference, Las Vegas, NV, October 1999.

[2] Johnson R. Jr, et. al. "Blind equalization using the constant modulus criterion: a overview," Proceedings of IEEE, Volume 86, October, 1988. 
[3] Satorius E.H. and Alexander S.T., "Channel equalization using adaptive lattice algorithm," IEEE Transactions on Communications, Volume COM-27, June 1979.

[4] Mansour D. and Gray A.H.G., "Unconstrainted Frequency-Domain Adaptive Filter," IEEE Transactions on Acoustics, Speech and Signal Processing, Vol. ASSP-30, October 1982.

[5] Lee J.C. and Un C.K., "Performance Analysis of frequency-Domain Block LMS Adaptive Filters," IEEE Transactions on Circuits and Systems, Vol. 36, February, 1989.

[6] Yang Y.G., "Fast blind equalization by using frequency domain block constant modulus algorithm," Proceeding of 38th Midwest Symposium on Circuit and Systems, 1995.

[7] Groginsky H.L. and Works G.A.., “A pipeline fast Fourier transform,” 1975.

[8] Moeller T.J. and Martinez D., "Field Programmable Gate Array Based Radar Front-end Digital Signal Processing," 1999.

[9] Xilinx Incorporated, “The Programmable Logic Data Book,” 1996.

[10] Xilinx Incorporated, "The role of distributed arithmetic in FPGA based signal processing,” 1996.

[11] New B., “A distributed arithmetic approach to design scalable DSP chips,” EDN, 1995.

[12] Goslin G.R., "Using Xifinx FPGAs to design custom digital signal processing devices,” In DSPX 1995 Technical Proceedings, 1995.

[13] Goslin G.R., "Implement DSP functions in FPGAs to reduce cost and boost performance," EDN, 1996. 